\title{
Dlabetes Versorgungs-Evaluation (DIVE) - eine nationale Initiative zur Qualitätssicherung in der diabetologischen Versorgung
}

\author{
„Dlabetes Versorgungs-Evaluation“ (DIVE) - a national quality assurance initiative \\ at physicians providing care for patients with diabetes
}

Autoren

\section{T. Danne ${ }^{1}$ M. Kaltheuner ${ }^{2,3}$ A. Koch ${ }^{4}$ S. Ernst ${ }^{4}$ W. Rathmann ${ }^{5}$ H.-J. Rüssmann $\dagger^{\dagger}$} P. Bramlage ${ }^{7}$ für die DIVE Studiengruppe

Institut

\author{
Kinder- und Jugendkrankenhaus „AUF DER BULT“, Hannover \\ 2 Gemeinschaftspraxis Kaltheuner - v. Boxberg, Leverkusen \\ winDiab GmbH, Düsseldorf \\ ${ }^{4}$ Institut für Biometrie, Medizinische Hochschule Hannover \\ Institut für Biometrie und Epidemiologie, Deutsches Diabetes Zentrum Düsseldorf \\ Schwerpunktpraxis Dr. Rüssmann, Dinslaken-Bruch \\ 7 Institut für Pharmakologie und präventive Medizin, Mahlow
}

\section{Diabetologie, \\ Versorgungsforschung \\ Schüsselwörter \\ $\checkmark$ Diabetes \\ Repräsentativität \\ Versorgungsforschung \\ Routinedaten \\ Keywords \\ diabetes \\ representativeness \\ health care research \\ routine data}

eingereicht 08.10.2012 akzeptiert $\quad 02.04 .2013$

\section{Bibliografie}

Dol $10.1055 / s-0033-1343144$ Dtsch Med Wochenschr 2013; 138: 934-939 - (c) Georg Thieme Verlag KG · Stuttgart · New York · ISSN 0012-0472

Korrespondenz Prof. Dr. med. Thomas Danne Kinder- und Jugendkrankenhaus „AUF DER BULT“

Janusz-Korczak-Allee 12

30173 Hannover

Tel. 0511/81153330

Fax 0511/81155344

eMail danne@hka.de

\section{Zusammenfassung}

$\checkmark$

Hintergrund: Die strukturierte Dokumentation von Patienten mit Diabetes mellitus ist aufgrund der Vielfalt der betroffenen Organsysteme mit Notwendigkeit zur fachgebietsübergreifenden Vernetzung der individuellen diagnostischen und therapeutischen Strategien von zunehmender Bedeutung. Dabei existieren viele Qualitätsinitiativen (z. B. SWEET, QS-DPV, EUBIROD) und mehrere Software-Systeme zur Erfassung Patienten-bezogener Daten (z. B. [DPV2] DIAMAX, DPV, EMIL, Qmax), die darüber hinaus Daten mit einer fast unüberschaubaren Zahl von Praxisverwaltungssystemen austauschen.

Methodik: Um dieser Problematik adäquat zu begegnen, wurde von diabetesDE in Zusammenarbeit mit Fachgesellschaften, Arztverbänden und Ärzten eine nationale Registerplattform ins Leben gerufen. Ziel von DIVE (DIabetes Versorgungs-Evaluation) ist es, ein nationales DiabetesRegister zu etablieren, das zentral die Daten von

\section{Hintergrund}

Diabetes ist mit mindestens 6 Mio. Betroffenen und einer Dunkelziffer von weiteren 2-3 Mio. eine der häufigsten Volkskrankheiten in Deutschland. Die Vereinten Nationen und die WHO haben das epidemieartige Ausmaß der chronischen Lebensstilkrankheiten (Diabetes, Herz-Kreislauf-, Krebs- und Lungenerkrankungen) anerkannt und zugestimmt, koordinierte Aktionen auf globaler Ebene vorzunehmen. Mitte September 2011 hat das Regionalbüro Europa der WHO einen Europäischen Aktionsplan zur Kontrolle und Bekämpfung der nichtübertragbaren Krankheiten veröffentlicht. Die Mitgliedstaaten der UN sind aufgefordert, bis $2013 \mathrm{Na}$ tionale Aktionspläne gegen nichtübertragbare Krankheiten aufzustellen. Dabei kommt der Entwicklung von krankheitsspezifischen Registern eine besondere Bedeutung zu.
Diabetes-Patienten in diabetologischen Schwerpunktpraxen in Deutschland zusammenführt und so für die Qualitätssicherung und Versorgungsforschung zugänglich macht.

Ergebnisse: Seit September 2011 wurden in DIVE durch 142 teilnehmende Ärzte insgesamt 84774 Patienten dokumentiert. Diese sind im Vergleich zu weiteren Registern vor allem im hausärztlichen Bereich durch ein deutlich fortgeschritteneres Krankheitsbild mit einer substantiellen Komorbidität gekennzeichnet.

Schlussfolgerung: Mit DIVE wurde eine nationale Registerplattform geschaffen, die wesentliche Handlungsfelder einer zu entwickelnden Nationalen Diabetes-Strategie - Epidemiologie, Diabetesregister, Versorgungsforschung, Versorgungsstrukturen und Qualitätssicherung - auf der Basis üblicher Programme zur Patientendokumentation der Praxisverwaltung in den Fokus rückt und zur Verbesserung der Versorgung von Patienten mit Diabetes beitragen wird.

Aufgrund der vielen existierenden Dokumentationssystemen wie der DPV2-Gesundheitsplattform (DPV $=$ Diabetes Patienten Verlaufsdokumentation) und dem dafür entwickelten Diabetes-Modul [DPV2] DIAMAX (axaris GmbH, Ulm), mit DPV (Universität Ulm) $[4,8]$, Qmax (GMC Systems mbH, Ilmenau) und EMIL (ITC, Marburg) und zentralen Datenerfassungen wie dem SWEET-Projekt (Better control in pediatric and adolescent diabetes: working to create Centres of Reference) [6], QS-DPV [2], winDIAB und FQSD entsteht die Notwendigkeit, Doppeleingaben von Patientendaten in unterschiedliche Dokumentationssysteme zu vermeiden. 
DIVE (DIabetes Versorgungs-Evaluation; www.dive-register.de) ist ein nationales Registerprojekt in Deutschland. Es zielt auf eine Qualitätssicherung in der Behandlung von ambulanten Patienten mit Diabetes mellitus in Schwerpunktpraxen und Klinikambulanzen in Deutschland unter Nutzung einer spezifischen strukturierten Dokumentationssoftware: Der Schwerpunkt liegt auf der Umsetzung der Therapieleitlinien der DDG, um Therapieadhärenz zu fördern und das Auftreten von Endorganschäden zu verhindern. Die Daten sollen Grundlage für eine nationale Diabetesstrategie sein. DIVE stellt eine gemeinsame Datenplattform zur Verfügung, die offen konzipiert ist und sich das Ziel gesetzt hat, Datenimporte aus verschiedenen Dokumentationssystemen ([DPV2] DIAMAX, DPV, EMIL, Qmax) zu ermöglichen, und verschiedene Datenbanken für die nationalen oder EU-weiten Diabetes-Initiativen zu bedienen. In der vorliegenden Arbeit wird die Methodik vorgestellt und die Patientenpopulation charakterisiert.

\section{Methoden}

DIVE ist eine nationale Registerplattform in Deutschland, an der sich Schwerpunktpraxen und Klinikambulanzen im gesamten Bundesgebiet beteiligen. Aktuell geht man dabei von etwa 693 Schwerpunktpraxen aus. Die Teilnehmer sind nicht prä-selektiert, sondern können sich für eine Teilnahme an DIVE beim Projektträger melden. Eine Teilnahme von mindestens 250 Ärzten, entsprechend etwa einem Drittel aller Praxen, wird angestrebt. Die Ethikkommission der Medizinischen Hochschule Hannover hat das Registerprotokoll begutachtet und mit Schreiben vom 25.8.2011 genehmigt [3].

\section{Patienten}

Von den Ärzten werden konsekutiv alle Patienten mit Diabetes mellitus aufgenommen, unabhängig vom Krankheitsstadium sowie der individuellen Therapiestrategie. Die Mindestanzahl beträgt 250 Patienten pro Praxis, die ihre Einwilligung schriftlich erklären müssen [3]. Daraus resultiert bei Teilnahme von 250 Praxen eine Zahl von mindestens 62500 Patienten. Eine Vollerhebung aller Patienten in der Praxis wird angestrebt und könnte die Fallzahl weiter erhöhen. Diese ist allerdings über die beschriebene Notwendigkeit eines Patienteneinverständnisses limitiert. Darüber hinaus werden keine weiteren Ein- oder Ausschlusskriterien definiert.

\section{Datenerfassung}

In Zusammenarbeit mit der Deutschen Diabetes-Gesellschaft (DDG), der Firma axaris und mit der Unterstützung von Lilly Deutschland wurde kürzlich eine Software entwickelt: [DPV2] DIAMAX. Hiermit soll eine einheitliche IT-Basis in der deutschen Diabetologie geschaffen werden. [DPV2] DIAMAX wurde bereits für das SWEET-Projekt [6] der International Society for Pediatric and Adolescent Diabetes (ISPAD) und das DLAN-Projekt (Diabetes Lokales Ausbildungsnetzwerk) [7] der DDG verwendet. Gleichzeitig werden die Ärzte in der systematischen Nutzung einer strukturierten Dokumentation ihrer Patienten mit Diabetes trainiert und profitieren für die Behandlungsabläufe ihrer Patienten von der standardisierten Dokumentation durch die Software. Damit in [DPV2] DIAMAX keine Daten doppelt dokumentiert werden müssen, können über die ebenfalls von axaris entwickelte Schnittstelle [DPV2] direktPVS eine Vielzahl an Parametern - weitaus mehr und auf eine komfortablere und somit praxistauglichere Weise als über die Möglichkeit eines BDT-Da-

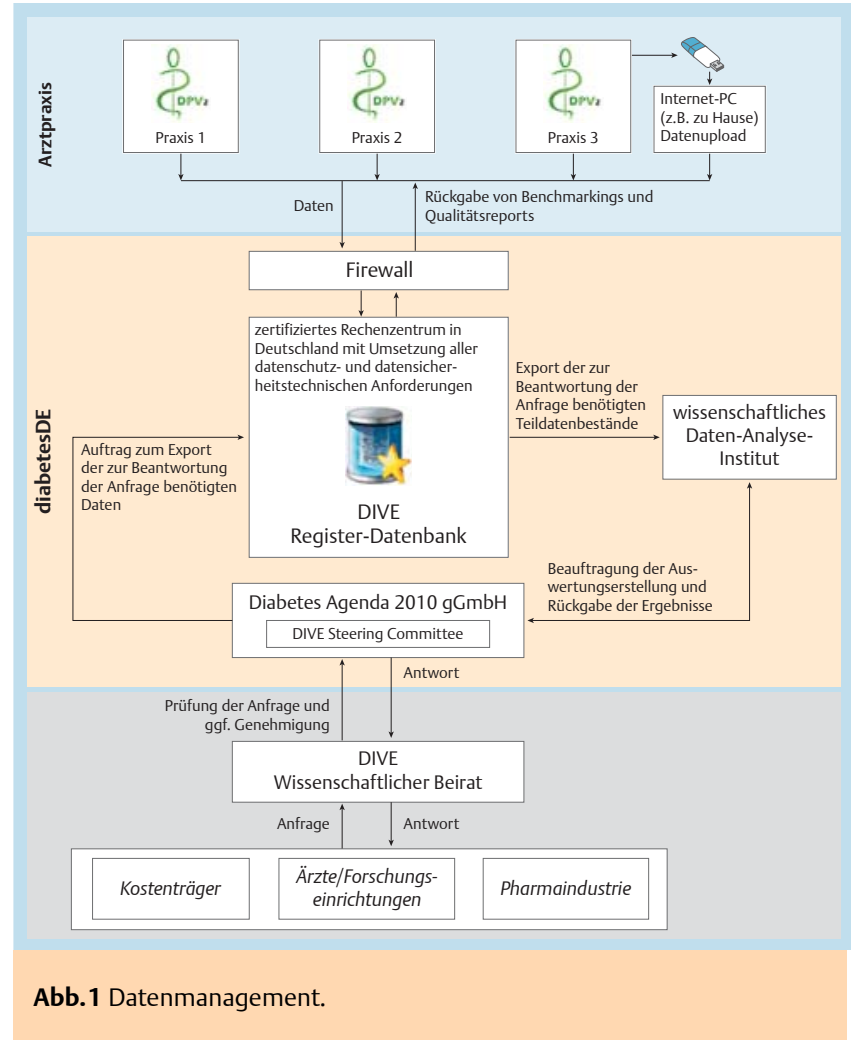

tenaustauschs - direkt aus den gängigsten Praxisverwaltungssystemen übernommen werden.

\section{Dokumentation}

Die Daten werden bei jedem Arztbesuch des Patienten erhoben und unterliegen keinem vorgegebenen Dokumentationsintervall. Sie umfassen eine Reihe von Variablen zur Struktur- und Prozessqualität in der Versorgung von Patienten mit Diabetes. Eine detaillierte Variablenliste wurde auf der Internetseite des Projekts veröffentlicht [3]. Dabei werden die Daten im Rahmen der ärztlichen Routine erhoben und sind, nach einer eher aufwendigen Anlaufphase, eine geringe zusätzliche Belastung für den dokumentierenden Arzt. Das Dokumentationsprogramm enthält Konsistenzprüfmodule, die eine fortlaufende Plausibilitätskontrolle der eingegebenen Daten ermöglichen.

\section{Datenübertragung}

Die pseudonymisierten Daten werden automatisch an einen gesicherten, ISO-zertifizierten Datenserver unter Verantwortung des Projektträgers diabetesDE bzw. deren Stellvertreter Diabetes Agenda $2010 \mathrm{GmbH}$ übertragen, die die Interessen des Projektträgers vertritt ( Abb.1). Dieser Server wird von der Firma Scanplus GmbH in Ulm betrieben. Die Daten verbleiben im Eigentum des teilnehmenden Arztes.

\section{Auswertungen}

Das Steering Committee entscheidet in Zusammenarbeit mit dem wissenschaftlichen Beirat über Anfragen zur Auswertung von DIVE. Diese Anfragen werden im Hinblick auf die notwendigen Auswertungen operationalisiert. Danach erteilt der Projektträger den Auftrag zur Auswertung der Fragestellungen. Die Ergebnisse der angeforderten Auswertungen werden nur in aggregierter, pseudonymisierter Form ohne Bezug zu konkreten einzelnen Praxen oder Patienten übergeben. Der komplette Rohdatensatz auf Patienten- und Praxisebene wird durch den 
Tab.1 Regionale Verteilung der teilnehmenden Praxen.

\begin{tabular}{|c|c|c|c|c|c|}
\hline \multirow[t]{2}{*}{$\begin{array}{l}\text { PLZ- } \\
\text { Gebiet }\end{array}$} & \multirow[t]{2}{*}{$\begin{array}{l}\text { Schwerpunktpraxen/ } \\
\text { Region nach DDG }\end{array}$} & \multicolumn{2}{|c|}{$\begin{array}{l}\text { Teilnehmende } \\
\text { Praxen }\end{array}$} & \multicolumn{2}{|c|}{ Patienten } \\
\hline & & $\mathbf{n}$ & $\%$ & $\mathbf{n}$ & $\%$ \\
\hline 0 & 44 & 4 & 2,8 & 1193 & 1,4 \\
\hline 1 & 49 & 11 & 7,8 & 3940 & 4,7 \\
\hline 2 & 73 & 10 & 7,0 & 3452 & 4,1 \\
\hline 3 & 80 & 17 & 12,0 & 10663 & 12,6 \\
\hline 4 & 82 & 25 & 17,6 & 17816 & 21,0 \\
\hline 5 & 78 & 26 & 18,3 & 12820 & 15,1 \\
\hline 6 & 83 & 17 & 12,0 & 10701 & 12,6 \\
\hline 7 & 77 & 10 & 7,0 & 3588 & 4,2 \\
\hline 8 & 64 & 11 & 7,8 & 7023 & 8,3 \\
\hline 9 & 63 & 11 & 7,8 & 13578 & 16,0 \\
\hline Gesamt & 693 & 142 & 100 & 84774 & 100 \\
\hline
\end{tabular}

Projektträger nicht an Dritte abgegeben. Die Daten verbleiben unter Verantwortung des Projektträgers und stehen damit langfristig auch über die vorliegende Erhebung hinaus für weitere wissenschaftliche Auswertungen zur Verfügung.

\section{Statistik}

Für die Ergebnisdarstellung erfolgte die biometrische Auswertung aller erhobenen Daten deskriptiv. Die Ergebnisse wurden tabellarisch und graphisch aufbereitet. Für stetige Variablen wurden Mittelwert, Standardabweichung, Median, Minimum und Maximum und für kategoriale Variablen die absoluten und relativen Häufigkeiten bestimmt. Alle statistischen Analysen wurden mit dem statistischen Software-Paket SAS 9.3 oder höher (SAS Institute, Cary, NC, USA) durchgeführt.

\section{Ergebnisse}

Patienteneinschluss und regionale Verteilung

Seit September 2011 wurden von 142 Ärzten bisher 84774 Patienten dokumentiert (Stand: März 2013). Diese stammen aus allen Bundesländern ( Tab.1, Abb.2) mit einem bisherigen Schwerpunkt auf den Postleitzahlenbereichen 4, 5 und 9. Von diesen Patienten haben 13995 einen Typ-1- und 61471 einen Typ-2-Diabetes, 232 Patienten leiden an Typ-3-Diabetes sowie 1683 Patienten an Gestationsdiabetes, bei 3 Patienten ist die genaue Diagnose unbekannt und 7393 Patienten sind ohne Angabe. Die mediane Zahl von Patienten pro Praxis beträgt 314 (Interquartilen-Range 154-568). In der Darstellung beschränken wir uns zunächst auf Patienten mit Typ-1- und Typ-2-Diabetes mit einem Alter von 18-90 Jahren $(\mathrm{n}=64$ 273).

\section{Patientencharakteristika}

Patienten mit Typ-1-Diabetes haben ein mittleres Alter von 47,1 $\pm 16,0$ Jahren mit einem Häufigkeitsgipfel zwischen 35 und 49 Jahren. Patienten mit Typ-2-Diabetes sind im Mittel 17 Jahre älter (64,6 $\pm 12,0$ Jahre) und in den meisten Fällen zwischen 65 und 80 Jahre alt. Insgesamt sind etwa 46,7\% Frauen, die sich in der Gruppe der Typ-1- und Typ-2-Diabetiker gleichmäßig verteilen.

Bei der Betrachtung der 18- bis 90-jährigen Patienten lag der $\mathrm{HbA}_{1 \mathrm{c}}$ zur Aufnahme in das Register bei $62,5 \pm 17,8 \mathrm{mmol} / \mathrm{mol}$ (7,9 $\pm 1,6 \%$ ) beim Typ-1-Diabetes und bei $57,7 \pm 16,5 \mathrm{mmol} / \mathrm{mol}$ (7,4 $\pm 1,5 \%$ ) beim Typ-2-Diabetes im Mittel vergleichsweise

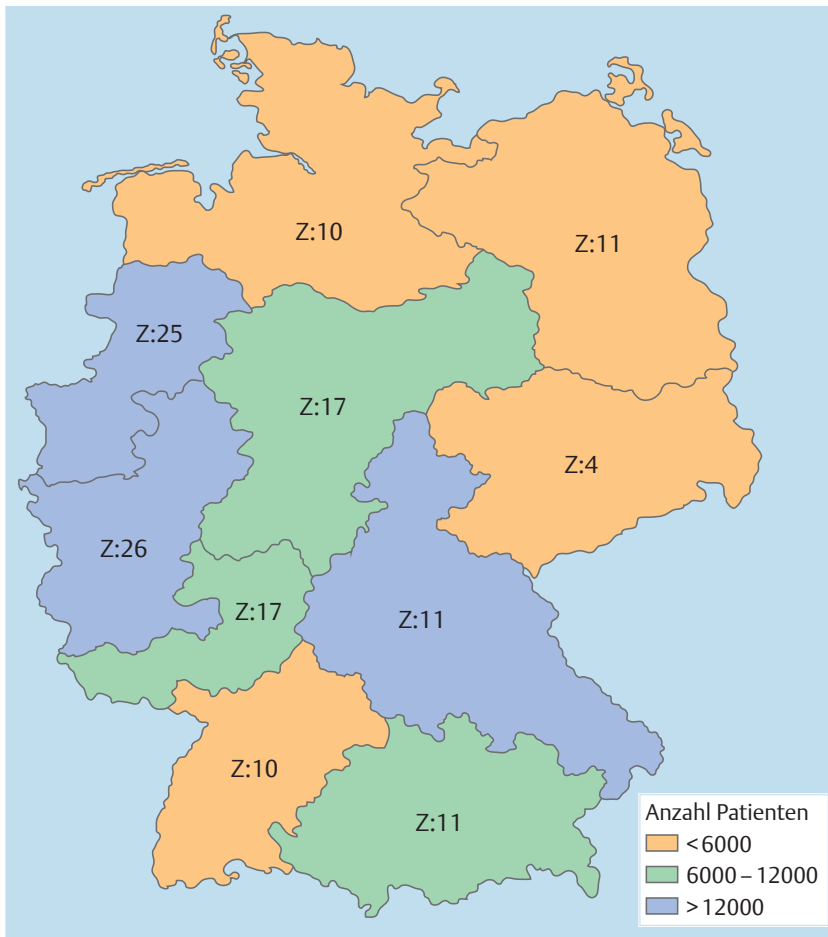

Abb.2 Regionale Verteilung der teilnehmenden Praxen (Zentren [Z]) und Anzahl der Patienten.

Tab.2 Patientencharakteristika und Laborwerte.

\begin{tabular}{|c|c|c|c|c|}
\hline & \multicolumn{2}{|c|}{$\begin{array}{l}\text { Typ-1-Diabetes } \\
\text { (n=11 777) }\end{array}$} & \multicolumn{2}{|c|}{$\begin{array}{l}\text { Typ-2-Diabetes } \\
\text { (n=52 496) }\end{array}$} \\
\hline & $\mathbf{n}$ & $\mathrm{MW} \pm \mathrm{SD} / \%$ & $\mathbf{n}$ & $\mathrm{MW} \pm \mathrm{SD} / \%$ \\
\hline Alter (Jahre) & 11777 & $47,1 \pm 16,1$ & 52496 & $64,6 \pm 12,0$ \\
\hline Frauen (\%) & 11777 & $5427(46,1 \%)$ & 52496 & $24596(46,9 \%)$ \\
\hline Raucher (\%) & 2777 & $595(21,4 \%)$ & 11058 & $1648(14,9 \%)$ \\
\hline Diabetes-Dauer (Jahre) & 8647 & $18,3 \pm 13,4$ & 35621 & $10,9 \pm 8,6$ \\
\hline$B M I\left(\mathrm{~kg} / \mathrm{m}^{2}\right)$ & 5768 & $26,7 \pm 5,1$ & 25275 & $32,1 \pm 6,7$ \\
\hline Bauchumfang $(\mathrm{cm})$ & 2228 & $93,1 \pm 14,8$ & 10661 & $107,3 \pm 18,0$ \\
\hline \multicolumn{5}{|l|}{ Laborwerte } \\
\hline $\begin{array}{l}\text { Gesamtcholesterin } \\
(\mathrm{mmol} / \mathrm{l})\end{array}$ & 4478 & $5,2 \pm 1,1$ & 22375 & $5,2 \pm 1,3$ \\
\hline $\begin{array}{l}\text { HDL-Cholesterin } \\
(\mathrm{mmol} / \mathrm{l})\end{array}$ & 4230 & $1,6 \pm 0,5$ & 20090 & $1,3 \pm 0,4$ \\
\hline $\begin{array}{l}\text { LDL-Cholesterin } \\
(\mathrm{mmol} / \mathrm{l})\end{array}$ & 4199 & $3,0 \pm 0,9$ & 19159 & $3,0 \pm 1,0$ \\
\hline Triglyceride (mmol/l) & 4335 & $1,5 \pm 2,0$ & 21040 & $2,2 \pm 2,1$ \\
\hline $\mathrm{HbA}_{1 \mathrm{c}}(\mathrm{mmol} / \mathrm{mol})$ & 8499 & $62,5 \pm 17,8$ & 37081 & $57,7 \pm 16,5$ \\
\hline$H b A_{1 c}(\%)$ & 8499 & $7,9 \pm 1,6$ & 37081 & $7,4 \pm 1,5$ \\
\hline $\begin{array}{l}\text { Nüchternblutzucker } \\
\text { (mmol/l) }\end{array}$ & 3655 & $9,2 \pm 4,6$ & 18680 & $8,3 \pm 3,5$ \\
\hline $\begin{array}{l}\text { Postprandiale } \\
\text { Glukose (mmol/l) }\end{array}$ & 2582 & $8,9 \pm 4,7$ & 10060 & $8,7 \pm 4,0$ \\
\hline
\end{tabular}

hoch (Tab.2). Der Nüchternblutzucker betrug $9,2 \pm 4,6$ $\mathrm{mmol} / \mathrm{l}$ (Typ-1-Diabetes) und 8,3 $\pm 3,5 \mathrm{mmol} / \mathrm{l}$ (Typ-2-Diabetes) sowie $8,9 \pm 4,7 \mathrm{mmol} / \mathrm{l}$ (Typ-1-Diabetes) und $8,7 \pm 4,0 \mathrm{mmol} / \mathrm{l}$ (Typ-2-Diabetes) für die postprandiale Glukose.

\section{Begleiterkrankungen und Risikofaktoren}

Tab.3 zeigt, dass die Patienten häufig Begleiterkrankungen und Diabetes-bedingte Komplikationen aufweisen. Insgesamt 
Tab.3 Begleiterkrankungen.

\begin{tabular}{lll|} 
& $\begin{array}{l}\text { Typ-1-Diabetes } \\
(\mathbf{n = 1 1} \text { 777) }\end{array}$ & $\begin{array}{l}\text { Typ-2-Diabetes } \\
\text { (n=52 496) }\end{array}$ \\
& $\%$ & $\%$ \\
\hline Koronare Herzerkrankung & 11,8 & 33,2 \\
\hline Schlaganfall & 2,9 & 6,9 \\
\hline Herzinfarkt & 1,6 & 4,9 \\
\hline pAVK & 11,9 & 24,4 \\
\hline $\begin{array}{l}\text { Diabetische Retinopathie } \\
\text { - Erblindung }\end{array}$ & 15,7 & 7,7 \\
\hline $\begin{array}{l}\text { Neuropathie } \\
\text { - Autonome }\end{array}$ & 0,2 & 0,1 \\
- Periphere & 9,8 & 8,8 \\
\hline pAVK=periphere arterielle Verschlusskrankheit & 57,5 \\
\hline
\end{tabular}

sind makrovaskuläre Kombinationen wie KHK und Schlaganfälle sowie mikrovaskuläre Komplikationen wie pAVK bei Typ-2Diabetes sehr viel häufiger als bei Typ-1-Diabetes.

\section{Pharmakotherapie}

Die meisten Patienten mit Typ-1-Diabetes erhalten kurz(87,8\%) und/oder langwirksames Insulin (66,3\%). Diese kommen bei Patienten mit Typ-2-Diabetes sehr viel seltener zum Einsatz. Hier werden vor allem Metformin $(51,1 \%)$ und Sulfonylharnstoffe $(14,1 \%)$ eingesetzt.

Kardiovaskuläre Risikofaktoren wie Dyslipidämie und Hypertonie werden bei Typ-2-Diabetes häufig therapiert ( Tab.4). Insgesamt ist bei den Patienten mit Typ-2-Diabetes zu sehen, dass diese ihr Therapieziel (Dyslipidämie und Hypertonie) seltener als Patienten mit Typ-1-Diabetes erreichen ( Abb.3). Beim Typ-1-Diabetes sind etwa $74 \%$ aller Patienten normoton, haben aber in einem hohen Prozentsatz erhöhte Lipide (69\%). $\mathrm{HbA}_{1 \mathrm{c}^{-}}$ Zielwerte werden in vielen Fällen nicht erreicht. Bei Typ-2-Diabetes zeigen sich insbesondere in Abgrenzung zum Typ-1-Diabetes häufigere Zielwerterreichung für den $\mathrm{HbA}_{1 \mathrm{c}}$.
Tab.4 Dokumentierte antidiabetische Medikation auf Patientenebene.

\begin{tabular}{|c|c|c|}
\hline & $\begin{array}{l}\text { Typ-1-Diabetes } \\
\text { (n=11 777) } \\
\text { n (\%) }\end{array}$ & $\begin{array}{l}\text { Typ-2-Diabetes } \\
\text { (n=52 496) } \\
n(\%)\end{array}$ \\
\hline Metformin & $772(6,6 \%)$ & $26838(51,1 \%)$ \\
\hline Sulfonylharnstoffe & - & $7379(14,1 \%)$ \\
\hline Glukosidasehemmer & - & $474(0,9 \%)$ \\
\hline DPP-4-Hemmer & - & $5559(10,6 \%)$ \\
\hline GLP-Analoga & - & $3262(6,2 \%)$ \\
\hline Kurzwirksame Insuline & $10345(87,8 \%)$ & 18607 (35,4\%) \\
\hline Langwirksame Insuline & $7847(66,6 \%)$ & $20412(38,9 \%)$ \\
\hline Mischinsulin & $189(1,6 \%)$ & $3795(7,2 \%)$ \\
\hline ACE-Hemmer & $2118(18,0 \%)$ & $14986(28,6 \%)$ \\
\hline$A T_{1}$-Blocker & $925(7,9 \%)$ & $7337(14,0 \%)$ \\
\hline Betablocker & $1331(11,3 \%)$ & $13898(26,5 \%)$ \\
\hline Diuretika & $834(7,1 \%)$ & $10046(19,1 \%)$ \\
\hline Calciumantagonisten & $837(7,1 \%)$ & $8497(16,2 \%)$ \\
\hline Statine / Fibrate & $1930(16,4 \%)$ & 15919 (30,3\%) \\
\hline Plättchenhemmer & $780(6,6 \%)$ & $7870(15,0 \%)$ \\
\hline $\begin{array}{l}\text { Keine dokumentierte } \\
\text { Medikation }\end{array}$ & $613(5,2 \%)$ & $1945(3,7 \%)$ \\
\hline Sonstige Medikationen & $6580(55,9 \%)$ & $27917(53,2 \%)$ \\
\hline
\end{tabular}

\section{Diskussion}

$\checkmark$

Mit DIVE wurde aktuell auf der Basis üblicher Programme zur Patienten- und Praxisverwaltung eine nationale Registerplattform ins Leben gerufen, die wesentliche Handlungsfelder der von der UN für jedes Land geforderten Nationalen DiabetesStrategie - Epidemiologie, Diabetesregister, Versorgungsforschung, Versorgungsstrukturen und Qualitätssicherung - adressiert. Sie ist direkte Konsequenz aus der 2006 von Kofi Annan formulierten Erkenntnis, „to do nothing is no longer an option“. Mit dem Aufbau der Server-Infrastruktur, der Installation der [DPV2] DIAMAX-Software in mehr als 142 diabetologischen Schwerpunktpraxen seit September 2011 und dem Einschluss von momentan bereits 84774 Patienten wurde die Grundlage

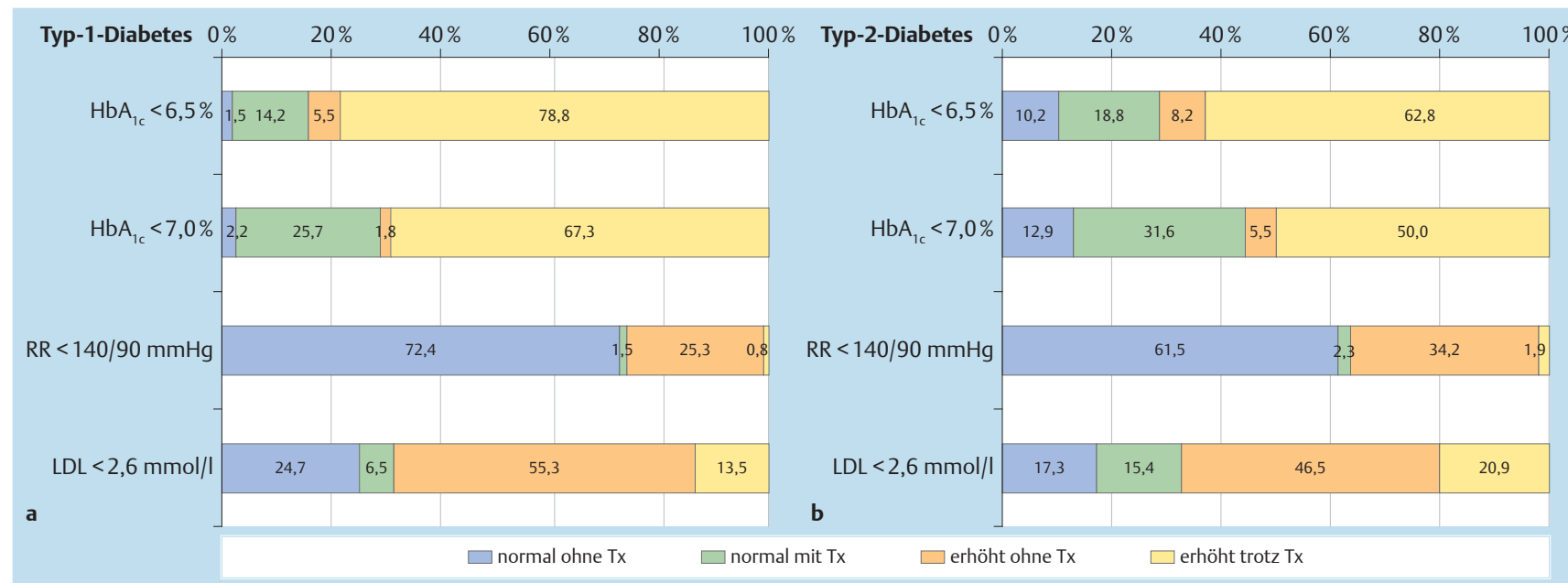

Abb. 3 Prozentualer Anteil der Patienten mit Typ-1-Diabetes (a) bzw. Typ-2 Diabetes (b), die bei Einschluss (1. dokumentierte Visite) die Therapieziele nach den Praxisleitlinien der Deutschen Diabetes Gesellschaft erreichen: RR = Blutdruck; (Zielblutdruck $\leq 140 / 90 \mathrm{mmHg}$ ); $\mathrm{LDL}=\mathrm{LDL}-\mathrm{Cholesterin} \mathrm{(<2,6} \mathrm{mmol/I);}$ $\mathrm{HbA}_{1 \mathrm{c}}=$ glykosyliertes Hämoglobin $\mathrm{A}_{1 \mathrm{c}}$ (Zielwert 6,5 bzw. 7,0\%); Tx = Behandlung. 
für ein Nationales Diabetesregister gelegt. Mit der vorgelegten Arbeit wird die Machbarkeit dieses Vorgehens aus Routinedaten belegt. DIVE soll nun durch eine longitudinale Weiterverfolgung der Daten und eine Ausdehnung des Registers auf die verschiedenen Versorgungsebenen langfristig weiterentwickelt werden.

Dazu ist es notwendig, dass die verschiedenen an der Versorgung von Patienten mit Diabetes beteiligten Fachgruppen und Verbände an einem Strang ziehen. Dafür ist mit der Einbeziehung der Deutschen Diabetes Gesellschaft (DDG), dem Verband der Diabetes-Beratungs- und Schulungsberufe in Deutschland e.V. (VDBD), dem Bundesverband Niedergelassener Diabetologen e.V. (BVND), der Arbeitsgemeinschaft niedergelassener diabetologisch tätiger Ärzte e.V. (AND), dem Wissenschaftlichen Institut der niedergelassenen Diabetologen (winDiab), dem Bundesverband der Diabetologen in Kliniken e.V. (BVDK) und einem Vertreter des Kompetenznetzwerkes Diabetes ein wichtiger erster Schritt getan. Weitere Unterstützung ist ausdrücklich erwünscht, sie kann nur zu einer Verbreiterung der Basis von Ärzten und Patienten beitragen und die Bedeutung dieser Plattform für die Patientenversorgung erhöhen.

Wie in Projekten dieser Art nicht zu vermeiden, verliefen die Anlaufphase und die Einbindung der Vielzahl von bestehenden Praxisverwaltungssystemen, aus denen Daten in DIVE exportiert werden können, nicht ohne Komplikationen. Viele dieser Themen konnten in direktem Kontakt mit dem teilnehmenden Arzt geklärt oder in zusätzlichen Vor-Ort-Terminen behoben werden. Trotzdem lernt das System auch weiterhin laufend aus dem Praxisalltag und sieht sich gewappnet, den sich verändernden Anforderungen an Patientendokumentation und Softwareumgebungen Rechnung zu tragen.

Ein weiteres wichtiges Thema ist die Repräsentativität und Vollständigkeit der übertragenen Daten. Der erste Punkt kann durch die zunehmende Zahl von Teilnehmern und die Bereitstellung von mehr Schnittstellen und damit der Anbindung von noch mehr Ärzten erreicht werden. Der zweite Punkt ist vor allem auch eine Problematik von besonderer Bedeutung für die Anlaufphase. Werden in einer Praxis bis zu 1000 Patienten oder mehr, neu mit dieser Software dokumentiert, entsteht ein erheblicher Mehraufwand, der bei angenommenem Zeitbedarf für die Dokumentation von $10 \mathrm{Mi}$ nuten pro Patient etwa 160 Stunden im Startquartal erreicht. Daher haben wir in der Anlaufphase von einer strikten Einhaltung der Übergabe aller Pflichtvariablen abgesehen, müssen dieses Thema aber im weiteren Verlauf nachjustieren, um den Ansprüchen an Datenqualität und Vollständigkeit Rechnung zu tragen. Langfristig hoffen wir mit diesem System den zusätzlichen Aufwand durch DIVE zu minimieren, da faktisch ausschließlich auf Routinedaten zurückgegriffen wird. Dabei zeigen sich bereits in dieser ersten Auswertung auch unerwartete Ergebnisse aus der täglichen Praxis, wie der nicht so seltene Off-label-Einsatz von oralen Antidiabetika bei Patienten mit Typ-1-Diabetes, der z.B. bei erheblicher Adipositas, bei Insulinresistenz und Kriterien des metabolischen Syndroms oder auch bei latentem Autoimmundiabetes im Erwachsenenalter (LADA) diskutiert wird.

\section{Vergleich mit anderen Registern}

DIVE konzentriert sich auf die Dokumentation von Patienten, die in diabetologischen Schwerpunktpraxen behandelt werden, andere zur Zeit laufende Projekte legen das Hauptaugenmerk dagegen auf die Versorgung von Diabetes-Patienten in der Primärversorgung, d.h. bei Fachärzten für Allgemeinmedizin oder hausärztlich tätigen Internisten. Exemplarisch seien die DiaRegis und DIG-Register genannt $[1,5]$. Beide beschränken sich auf die Erfassung der Daten von Patienten mit Typ-2-Diabetes. Das mittlere Alter lag für die Patienten der DIG-Studie 2,4 Jahre unter dem der bisher in DIVE registrierten Patienten, hingegen waren die in DiaRegis eingeschlossenen Teilnehmer im Mittel etwas älter als die DIVE-Patienten mit Typ-2-Diabetes. Für alle Projekte wurde ein nahezu übereinstimmender Frauenanteil um die $47 \%$ errechnet. Die mittlere Diabetes-Dauer war in DIVE mit 10,1 Jahren deutlich höher als in DiaRegis (5,5 Jahre) und DIG (8,4 Jahre). Der gefundene mittlere $\mathrm{HbA}_{1 \mathrm{c}}$-Wert war in der DIG-Studie mit 7,0\% deutlich niedriger als in DiaRegis (7,4\%) und DIVE (8,2\%). Dabei muss man davon ausgehen, dass es auch zwischen den Teilnehmern innerhalb eines Registers erhebliche Unterschiede gibt. So lag der mittlere Zentrums$\mathrm{HbA}_{1 \mathrm{c}}$ im kinderdiabetologischen SWEET-Projekt im „besten“ europäischen Zentrum bei 7,6, im „schlechtesten“ bei 9,2\% [9]. Der Nüchternblutzucker war bei den DIG-Patienten $(7,6 \mathrm{mmol} / \mathrm{l})$ und bei den DiaRegis-Teilnehmern (7,9 mmol/l) besser eingestellt als in $\operatorname{DIVE}(8,2 \mathrm{mmol} / \mathrm{l})$. Für die postprandiale Glukose wurde ein umgekehrtes Verhältnis gefunden, hier hatten die Patienten in DIVE und DIG vergleichbare Werte (9,2 mmol/l), für DiaRegis wurde ein Mittelwert von $10,3 \mathrm{mmol} / \mathrm{l}$ errechnet.

Im Unterschied dazu waren die Werte für Gesamt-, HDL- und LDLCholesterin in den drei Projekten nahezu identisch. In Bezug auf die Begleiterkrankungen wurden sowohl mikro- als auch makrovaskuläre Komplikationen (Myokardinfarkt, KHK, Schlaganfall, pAVK, periphere Neuropathie, Retinopathie etc.) bei den in DIVE erfassten Typ-2-Diabetikern häufiger dokumentiert als bei den Teilnehmern in DIG und DiaRegis. Insgesamt zeigt dies die Limitation bestehender Register und die Notwendigkeit einer longitudinalen Verfolgung, wie sie im DIVE-Projekt möglich wird.

In der Gesamtschau zeigen die in DIVE aufgenommenen Typ-2-Diabetiker ein deutlich weiter fortgeschrittenes Krankheitsbild als die in den beiden anderen Projekten dokumentierten Teilnehmer. Dies ist sicherlich auf die im Schnitt längere Erkrankungsdauer, aber auch auf die Tatsache zurückzuführen, dass die Zuweisung zu einer auf die Behandlung des Diabetes spezialisierten Praxis im allgemeinen erst dann erfolgt, wenn die Schwere der Erkrankung, die Anzahl der Komplikationen und die dadurch bedingte erhöhte Komplexität der Behandlung dies geboten erscheinen lassen.

Als mögliche Limitation der Erhebung kann die bislang fehlende Vernetzung von Diabetologen und Hausärzten wahrgenommen werden. Diese ist grundsätzlich gewünscht, übersteigt aber zum jetzigen Zeitpunkt die Möglichkeiten des DIVE-Projektes. Daher ist eine langfristige unabhängige Förderung des DIVE-Projektes wünschenswert, die darüber hinaus eine Erweiterung der Datenbasis möglich machen könnte.

\section{Schlussfolgerung}

Mit DIVE wurde zunächst für Diabetes-Schwerpunktpraxen eine nationale Registerplattform geschaffen, der die Machbarkeit eines wesentlichen Handlungsfelds der Nationalen Diabetes-Strategie - Epidemiologie, Diabetesregister, Versorgungsforschung, Versorgungsstrukturen und Qualitätssicherung - in den Fokus rückt und auf der Basis üblicher Programme zur Patientendokumentation in der Praxisverwaltung zur Verbesserung der Versorgung von Patienten mit Diabetes beitragen wird. 


\section{Konsequenz für Klinik und Praxis}

- DIVE ist ein nationales Register von Patienten in diabetologischen Schwerpunktpraxen, das eine möglichst flächendeckende Dokumentation von Patienten mit Diabetes zum Ziel hat. - Die Daten werden dazu beitragen, die Versorgung von Patienten mit Diabetes in Deutschland zu verbessern und Anregungen für Optimierungen aus dem Praxisalltag abzuleiten.

- Für die beteiligten Ärzte besteht die Möglichkeit einer für die eigene Praxis spezifischen Auswertung im Vergleich mit den Kollegen, um so Handlungsfelder für die Verbesserung der Versorgung zu erkennen.

\section{DIVE-Studiengruppe}

\section{Steering Committee}

Thomas Danne (Hannover), Dietrich Garlichs (Berlin), Peter Bramlage (Mahlow), Matthias Kaltheuner (Leverkusen), Holger Fleischmann (Berlin)

\section{Wissenschaftlicher Beirat}

Thomas Danne (Hannover), Thomas Haeger (Kiel), Michael Jecht (Berlin), Thomas Kipp (St. Ingbert), Wolfgang Rathmann (Düsseldorf), Hans-Martin Reuter (Jena), Nikolaus Scheper (Marl), Erhard G. Siegel (Heidelberg), Dietmar Weber (Köln), Christian Berg (Essen)

\section{Weitere Projektmitarbeiter}

Stefanie Ernst (Hannover), Armin Koch (Hannover), Berndt von Stritzky (Berlin), Michael Volk (Ulm)

Danksagung: DIVE ist eine nationale Qualitätsinitiative von diabetesDE unter Beteiligung der Deutschen Diabetes Gesellschaft (DDG), dem Verband der Diabetes-Beratungs- und Schulungsberufe in Deutschland e.V. (VDBD), dem Bundesverband Niedergelassener Diabetologen e.V. (BVND), der Arbeitsgemeinschaft niedergelassener diabetologisch tätiger Ärzte e.V. (AND), dem Wissenschaftlichen Institut der niedergelassenen Diabetologen (winDiab) und dem Bundesverband der Diabetologen in Kliniken e.V. (BVDK). [DPV2]-DIAMAX ist eine von der Firma axaris - software \& systeme $\mathrm{GmbH}$ in Kooperation mit der DDG und Unterstützung der Firma Lilly Deutschland GmbH entwickelte Software. DIVE wurde durch eine Anschubfinanzierung der Firma Sanofi Aventis Deutschland $\mathrm{GmbH}$ und perspektivisch weitere Förderer finanziell ermöglicht.

Widmung: Diese Publikation ist Herrn Dr. Heinz-Jürgen Rüssmann (Dinslaken-Bruch) gewidmet, der im Wissenschaftlichen Beirat die Arbeitsgemeinschaft niedergelassener diabetologisch tätiger Ärzte e.V. (AND) vertrat und am 2. Juni 2012 unerwartet und viel zu früh starb.

Autorenerklärung: Die Studie wurde von der Firma Sanofi unterstützt. TD, MK, AK, SE, WR erklären, dass sie keine finanzielle Verbindung mit einer Firma haben, deren Produkt in diesem Beitrag eine Rolle spielt (oder mit einer Firma, die ein Konkurrenzprodukt vertreibt). PB erklärt, dass er Forschungsunterstützung sowie Honorare für Beratung und Vorträge der Firmen Bristol Myers Squibb, Astra Zeneca, Novartis und Sanofi erhalten hat.

\section{Abstract}

Dlabetes Versorgungs-Evaluation (DIVE) - a national quality assurance initiative at physicians providing care for patients with diabetes $\nabla$

Background: Due to the variety of affected organ systems, necessitating a multidisciplinary and interconnected approach in deciding on individual diagnostic and therapeutic strategies, a structured documentation of data for patients suffering from diabetes mellitus is steadily gaining importance. Towards this purpose, multiple quality initiatives (e.g. SWEET, QS-DPV, EUBIROD etc.) as well as several software systems (e.g. [DPV2] DIAMAX, DPV, EMIL, Qmax etc.) have been developed to capture patientrelated data. This is further complicated by the necessity to exchange data with a large variety of doctor's office administration systems.

Methods: To address this complex of issues, DiabetesDE in cooperation with several societies, doctor's associations and prospective end users launched a national register platform. DIVE (Diabetes Care Evaluation) is aimed at establishing a national diabetes register to centrally capture data from diabetes patients being treated by diabetology specialists in Germany, thus making them available for quality assurance and health services research.

Results: Since September 2011, 142 so far participating doctors have documented data for 84,774 patients. Compared to patients treated by general practitioners, persons under specialist care show a more advanced clinical picture with substantial co-morbidity.

Conclusion: DIVE provides a national platform which will address essential fields of activity with regard to the development of a national diabetes strategy - epidemiology, diabetes registry, health care research, quality assurance - based on usual office administration systems, thus contributing to the improvement of care and treatment for patients suffering from diabetes.

\section{Literatur}

1 Bramlage P, Binz C, Krekler $M$ et al. Diabetes Treatment Patterns and Goal Achievement in primary diabetes care (DiaRegis). Cardiovasc Diabetol 2010; 9: 54

2 Diabetologie Ap. Statement Qualitätssicherung in der Pädiatrischen Diabetologie. Monatsschr Kinderheilkd 1995; 143: 1146-1149

3 DIVE Studiengruppe. Registerprotokoll Diabetes Versorgungs-Evaluation (DIVE). 2011; http://www.dive-register.de/data/Protokoll_ DIVE_20110916\%20110920final.pdf

4 Grabert M, Schweiggert F, Holl RW. A framework for diabetes documentation and quality management in Germany: 10 years of experience with DPV. Comput Methods Programs Biomed 2002; 69: 115121

5 Hanefeld M, Koehler C, Gallo S et al. Impact of the individual components of the metabolic syndrome and their different combinations on the prevalence of atherosclerotic vascular disease in type 2 diabetes: the Diabetes in Germany (DIG) study. Cardiovasc Diabetol 2007; 6: 13

6 ISPAD. Better control in Pediatric and Adolescent diabetes: working to create Centres of Reference. 2012; http://sweet-project.eu

7 Jecht M, Landgraf R. Qualitätssicherung in der Diabetologie. Der Diabetologe 2011; 7: 119-132

8 Schutt M, Kern W, Krause $U$ et al. Is the frequency of self-monitoring of blood glucose related to long-term metabolic control? Multicenter analysis including 24,500 patients from 191 centers in Germany and Austria. Exp Clin Endocrinol Diabetes 2006; 114: 384-388

9 ISPAD. Better control in pediatric and adolescent diabetes: working to create Centres of Reference 2012. http://sweet-project.eu 